\title{
ON THE LITTLE SECONDARY BRUHAT ORDER*
}

\author{
ROSRIO FERNANDES ${ }^{\dagger}$, HENRIQUE F. DA CRUZ , AND DOMINGOS SALOMÃO $^{\S}$
}

\begin{abstract}
Let $R$ and $S$ be two sequences of positive integers in nonincreasing order having the same sum. We denote by $\mathcal{A}(R, S)$ the class of all $(0,1)$-matrices having row sum vector $R$ and column sum vector $S$. Brualdi and Deaett (More on the Bruhat order for (0,1)-matrices, Linear Algebra Appl., 421:219-232, 2007) suggested the study of the secondary Bruhat order on $\mathcal{A}(R, S)$ but with some constraints. In this paper, we study the cover relation and the minimal elements for this partial order relation, which we call the little secondary Bruhat order, on certain classes $\mathcal{A}(R, S)$. Moreover, we show that this order is different from the Bruhat order and the secondary Bruhat order. We also study a variant of this order on certain classes of symmetric matrices of $\mathcal{A}(R, S)$.
\end{abstract}

Key words. (0,1)-matrices, Interchanges, Secondary Bruhat order, Minimal elements.

AMS subject classifications. 05B20, 06A07.

1. Introduction. In the last decades, matrices whose entries are just zeros and ones, the $(0,1)$-matrices, have been heavily studied. They play an important role in mathematical optimization, combinatorics, matrix theory, graph theory, etc.. Let $m$ and $n$ be two positive integers. Let $R=\left(r_{1}, \ldots, r_{m}\right)$ and $S=\left(s_{1}, \ldots, s_{n}\right)$ be two fixed sequences of positive integers in nonincreasing order such that

$$
r_{1}+\cdots+r_{m}=s_{1}+\cdots+s_{n}
$$

In this paper, we will focus on the class $\mathcal{A}(R, S)$ of all $m$-by- $n(0,1)$-matrices with row sum vector $R$ and column sum vector $S$. When $m=n$ and the vectors $R$ and $S$ have all coordinates equal to a positive integer $k$, we denote $\mathcal{A}(R, S)$ by $\mathcal{A}(n, k)$.

An important theorem due to Ryser $([3,17,18])$ states that if the class $\mathcal{A}(R, S)$ is nonempty, then any single matrix $A \in \mathcal{A}(R, S)$ can generate the entire class $\mathcal{A}(R, S)$ by simple transformations called interchanges. An interchange replaces one of the 2-by-2 submatrices

$$
I_{2}=\left[\begin{array}{ll}
1 & 0 \\
0 & 1
\end{array}\right] \quad \text { or } \quad L_{2}=\left[\begin{array}{ll}
0 & 1 \\
1 & 0
\end{array}\right]
$$

by the other, [3]. When we replace a submatrix $I_{2}$ by $L_{2}$, we say that we perform an $I_{2} \rightarrow L_{2}$ interchange. Similarly, when we replace a submatrix $L_{2}$ by $I_{2}$ we say that we perform an $L_{2} \rightarrow I_{2}$ interchange.

In 2004, Brualdi and Hwang generalized the classical Bruhat order of $S_{n}$, the symmetric group of degree $n$ to any class $\mathcal{A}(R, S)[2]$. Doing this they gave rise to two distinct partial order relations on $\mathcal{A}(R, S)$. In the

\footnotetext{
${ }^{*}$ Received by the editors on April 3, 2020. Accepted for publication on December 11, 2020. Handling Editor: Geir Dahl. Corresponding Author: Henrique F. da Cruz.

${ }^{\dagger}$ CMA and Departamento de Matemática da Faculdade de Ciências Tecnologia, Universidade Nova de Lisboa, $2829-516$ Caparica, Portugal. (mrff@fct.unl.pt). Partially supported by the Fundação para a Ciência e a Tecnologia through the project UID/MAT/04721/2019.

${ }^{\ddagger}$ Departamento de Matemática da Universidade da Beira Interior, Rua Marquês D’Avila and Bolama, 6201-001 Covilhã, Portugal (hcruz@ubi.pt). Partially supported by the Fundação para a Ciência e a Tecnologia through the project UIDB/MAT/00212/2020.

$\S$ Universidade Mandume ya Ndemufayo, Escola Superior Pedagógica do Namibe, Angola (d.salomao@ubi.pt).
} 
last 16 years, many research have focused on several topics of these two partial order relations: conjectures [15], minimal elements [2, 4, 16], coincidence [12], chains and antichains [9, 10, 20, 21], restrictions of the Bruhat order on subclasses of $\mathcal{A}(R, S)[8,11]$, or extensions of one of these orders to other classes of matrices distinct of $\mathcal{A}(R, S)[5,6,7,13,14]$.

In [4], Brualdi and Deaett ended their paper with a list of several open problems on these partial order relations. One of those problems is the study of some constraints in one of these orders on $\mathcal{A}(R, S)$, and this is the main purpose of this paper. The paper is organized as follows: In Section 2, we make a brief description of the two partial orders defined on $\mathcal{A}(R, S)$ and we formally introduce the new partial order, the little secondary Bruhat order, which is the focus of our study. In Section 3, we characterize the cover relation for the little secondary Bruhat order and we show that this order does not coincide with the other two orders on $\mathcal{A}(R, S)$. In Section 4, we characterize the minimal matrices for the little secondary Bruhat order on $\mathcal{A}(n, 2)$, and in Section 5 we study it on $\mathcal{A}(2 k, k)$. In Section 6 , we see the restriction of this order to certain classes of symmetric $(0,1)$-matrices. Finally, in Section 7 we give some conclusions.

Here and throughout, we will denote by:

- $J_{m, n}$ or simply by $J$ the $m$-by- $n$ matrix whose entries are all equal to one;

- $O_{m, n}$ or simply by $O$ the $m$-by- $n$ null matrix;

- $I_{n}$ the identity matrix of order $n$;

- $L_{n}$ the permutation matrix of order $n$ with 1's on the antidiagonal;

- $A\left[\left\{i_{1}, \ldots, i_{r}\right\} ;\left\{j_{1}, \ldots, j_{s}\right\}\right]$ the submatrix of the $m$-by- $n$ matrix $A$ indexed by rows $i_{1}, \ldots, i_{r}$ and columns $j_{1}, \ldots, j_{s}$. If $\left\{i_{1}, \ldots, i_{r}\right\}=\left\{j_{1}, \ldots, j_{s}\right\}$, we simply write $A\left[\left\{i_{1}, \ldots, i_{r}\right\}\right]$.

2. Bruhat orders on $\mathcal{A}(R, S)$. For any $m$-by- $n$ real matrix $A=\left[a_{i, j}\right]$, let $\Sigma_{A}$ denote the $m$-by- $n$ matrix whose $(r, s)$-entry, with $1 \leq r \leq m, 1 \leq s \leq n$, is

$$
\sigma_{r, s}(A)=\sum_{i=1}^{r} \sum_{j=1}^{s} a_{i j}, \quad 1 \leq r \leq m, 1 \leq s \leq n .
$$

Brualdi and Hwang, in [2], extended the Bruhat order from the symmetric group of order $n$ to any nonempty class $\mathcal{A}(R, S)$. They do it using the matrices $\Sigma$. Given $A_{1}, A_{2} \in \mathcal{A}(R, S)$ we say that $A_{1}$ precedes $A_{2}$ in the Bruhat order, written $A_{1} \preceq_{B} A_{2}$, provided, in the entrywise order, $\Sigma_{A_{1}} \geq \Sigma_{A_{2}}$. Throughout that paper, [2], another partial order relation was mentioned but not formally defined. This second partial order, called the secondary Bruhat order, was defined a few years later by Brualdi and Deaett in [4]. Given $A_{1}, A_{2} \in \mathcal{A}(R, S)$ we say that $A_{1}$ precedes $A_{2}$ in the secondary Bruhat order, written $A_{1} \preceq \widehat{B} A_{2}$, provided $A_{1}$ can be obtained from $A_{2}$ by a sequence of $L_{2} \rightarrow I_{2}$ interchanges.

These two partial orders are both generalizations of the Bruhat order on the symmetric group $S_{n}$, and they have been intensively investigated in the recent years. It is straightforward to verify that if $A_{1}, A_{2} \in$ $\mathcal{A}(R, S)$ and $A_{1} \preceq_{\widehat{B}} A_{2}$, then $A_{1} \preceq_{B} A_{2}$, that is, the Bruhat order is a refinement of the secondary Bruhat order, but in general, the Bruhat order and the secondary Bruhat order do not coincide on $\mathcal{A}(R, S)$. However, Brualdi and Deaett proved, in [4], that they coincide on $\mathcal{A}(n, 2)$ as it happens on $\mathcal{A}(n, 1)$. Moreover, with an example, they showed that the two orders are distinct on $\mathcal{A}(6,3)$. In the same paper, Brualdi and Deaett suggested the study of another partial order relation on $\mathcal{A}(R, S)$ which is the secondary Bruhat order with some constraints. We call this new partial order relation the little secondary Bruhat order. Let $A_{1}, A_{2} \in \mathcal{A}(R, S)$. We say that $A_{1}$ precedes $A_{2}$ in the little secondary Bruhat relation provided $A_{1}=A_{2}$ or 
$A_{1}$ can be obtained from $A_{2}$ by a sequence of $L_{2} \rightarrow I_{2}$ where in each such interchange, the submatrix $L_{2}$ is formed by two consecutive rows.

If $A_{1}$ precedes $A_{2}$ in the little Bruhat order, then it is immediate to prove that $A_{1} \preceq_{B} A_{2}$. So, using the fact that the Bruhat order is antisymmetric we can prove that the little secondary Bruhat relation is a partial order. Thus, if $A_{1}$ precedes $A_{2}$ in the little secondary Bruhat order, then we write $A_{1} \preceq_{B^{\prime}} A_{2}$. The main purpose of this paper is to present some results on the little secondary Bruhat order.

Let $(X, \leq)$ be a finite partially ordered set. For $a, b \in X$, if $a \neq b$ and $a \leq b$, then we write $a<b$. We say $b$ covers $a$ if $a<b$ and there is not $d \in X$ with $a<d<b$.

The cover relation is an important subject of this work. We recall here the characterization of the cover relation in the secondary Bruhat order on $\mathcal{A}(R, S)$ [4].

TheOrem 2.1 ([4]). Let $A=\left[a_{s, v}\right] \in \mathcal{A}(R, S)$ and $A[\{i, j\} ;\{k, l\}]=L_{2}$. Let $A^{\prime}$ be the matrix obtained from $A$ by the $L_{2} \rightarrow I_{2}$ interchange that replaces the submatrix $A[\{i, j\} ;\{k, l\}]=L_{2}$ with $I_{2}$. Then $A$ covers $A^{\prime}$ in the secondary Bruhat order on $\mathcal{A}(R, S)$ if and only if

1. $a_{p, k}=a_{p, l}$, for $i<p<j$;

2. $a_{i, q}=a_{j, q}$, for $k<q<l$;

3. If $a_{p, k}=a_{i, q}=0$, then $a_{p, q}=0$, for $i<p<j$, and $k<q<l$;

4. If $a_{p, k}=a_{i, q}=1$, then $a_{p, q}=1$, for $i<p<j$, and $k<q<l$.

3. The cover relation. In this section, we will present some results on the little secondary Bruhat order. We will characterize its cover relation and we will show that the secondary Bruhat order and the little secondary Bruhat order do not coincide on $\mathcal{A}(n, 2)$ nor on $\mathcal{A}(n, 1)$.

Bearing in mind the definitions it is easy to conclude that if $A, D \in \mathcal{A}(R, S)$ and $A \preceq{ }_{B^{\prime}} D$ then $A \preceq \widehat{B} D$. So, we can ask if these two partial order relations coincide on $\mathcal{A}(R, S)$. For this answer, we start with the following proposition:

Proposition 3.1. Let $D \in \mathcal{A}(R, S), i, j \in\{1, \ldots, m\}$ and $k, l \in\{1, \ldots, n\}$ such that $i+1<j$ and $D[\{i, j\} ;\{k, l\}]=L_{2}$. Let $A$ be the matrix obtained from $D$ by the interchange $D[\{i, j\} ;\{k, l\}] \longrightarrow I_{2}$. If $D$ covers $A$ in the secondary Bruhat order, then $A$ and $D$ are incomparable elements in the little secondary Bruhat order.

Proof. We know that if $D \prec_{B^{\prime}} A$, then $D \prec_{\widehat{B}} A$, which is impossible because $D$ covers $A$ in the secondary Bruhat order.

Assume that $A \preceq B^{\prime} D$. If $A \neq D$, then we can obtain $A$ from $D$ by a sequence of $L_{2} \longrightarrow I_{2}$ interchanges where in each such interchange, $L_{2}$ is a submatrix formed by two consecutive rows. If $i+1<j$, then there is at least one matrix $D^{\prime} \in \mathcal{A}(R, S)$ such that

$$
A \prec_{B^{\prime}} D^{\prime} \prec_{B^{\prime}} D \text {. }
$$

This implies that $A \prec_{\widehat{B}} D^{\prime} \prec_{\widehat{B}} D$, which is impossible because $D$ covers $A$ in the secondary Bruhat order.

So, $A$ and $D$ are incomparable elements in the little secondary Bruhat order.

Now we can prove that the secondary Bruhat order and the little secondary Bruhat order do not coincide on $\mathcal{A}(n, 1)$ nor on $\mathcal{A}(n, 2)$. 
Let

$$
P=\left[\begin{array}{lll}
0 & 1 & 0 \\
0 & 0 & 1 \\
1 & 0 & 0
\end{array}\right] \text { and } Q=\left[\begin{array}{ccc}
1 & 0 & 0 \\
0 & 0 & 1 \\
0 & 1 & 0
\end{array}\right] \text {. }
$$

If $Q$ is obtained from $P$ by the $P[\{1,3\} ;\{1,2\}]=L_{2} \longrightarrow I_{2}$ interchange, then $Q \preceq_{\widehat{B}} P$. Moreover, using Theorem 2.1 we can conclude that $P$ covers $Q$ in the secondary Bruhat order. Therefore, by Proposition 3.1, $P$ and $Q$ are incomparable elements in the little Bruhat order.

So, if we consider the matrices

$$
P_{1}=\left[\begin{array}{cc}
P & O_{3, n-3} \\
O_{n-3,3} & I_{n-3}
\end{array}\right] \text { and } Q_{1}=\left[\begin{array}{cc}
Q & O_{3, n-3} \\
O_{n-3,3} & I_{n-3}
\end{array}\right]
$$

then we conclude that the secondary Bruhat order and the little secondary Bruhat order do not coincide on $\mathcal{A}(n, 1)$, for all $n \geq 3$.

Consider the following matrices of $\mathcal{A}(3,2)$ :

$$
D_{1}=\left[\begin{array}{lll}
0 & 1 & 1 \\
1 & 1 & 0 \\
1 & 0 & 1
\end{array}\right] \quad \text { and } A_{1}=\left[\begin{array}{lll}
1 & 0 & 1 \\
1 & 1 & 0 \\
0 & 1 & 1
\end{array}\right] \text {. }
$$

If $A_{1}$ is obtained from $D_{1}$ by the $D_{1}[\{1,3\} ;\{1,2\}]=L_{2} \longrightarrow I_{2}$ interchange, then $A_{1} \preceq_{\widehat{B}} D_{1}$. Moreover, using Theorem 2.1 we can conclude that $D_{1}$ covers $A_{1}$ in the secondary Bruhat order. Therefore, by Proposition 3.1, $D_{1}$ and $A_{1}$ are incomparable elements in the little Bruhat order.

Now consider the following matrices of $\mathcal{A}(4,2)$ :

$$
D_{2}=\left[\begin{array}{llll}
0 & 1 & 1 & 0 \\
0 & 0 & 1 & 1 \\
1 & 0 & 0 & 1 \\
1 & 1 & 0 & 0
\end{array}\right] \text { and } A_{2}=\left[\begin{array}{cccc}
1 & 0 & 1 & 0 \\
0 & 0 & 1 & 1 \\
0 & 1 & 0 & 1 \\
1 & 1 & 0 & 0
\end{array}\right] \text {. }
$$

If $A_{2}$ is obtained from $D_{2}$ by the $D_{2}[\{1,3\} ;\{1,2\}]=L_{2} \longrightarrow I_{2}$ interchange, then $A_{2} \preceq_{\widehat{B}} D_{2}$. Moreover, using Theorem 2.1 we can conclude that $D_{2}$ covers $A_{2}$ in the secondary Bruhat order. Therefore, by Proposition 3.1 we get $D_{2}$ and $A_{2}$ are incomparable elements in the little Bruhat order.

So, if we consider the matrices

$$
D_{i}^{\prime}=\left[\begin{array}{ccccc}
D_{i} & O & O & \ldots & O \\
O & J_{2} & O & \ldots & O \\
O & O & J_{2} & \ldots & O \\
\vdots & \vdots & \vdots & \ddots & \vdots \\
O & O & O & \ldots & J_{2}
\end{array}\right] \text { and } A_{i}^{\prime}=\left[\begin{array}{ccccc}
A_{i} & O & O & \ldots & O \\
O & J_{2} & O & \ldots & O \\
O & O & J_{2} & \ldots & O \\
\vdots & \vdots & \vdots & \ddots & \vdots \\
O & O & O & \ldots & J_{2}
\end{array}\right]
$$

for $i=1,2$, then we conclude that the secondary Bruhat order and the little secondary Bruhat order do not coincide on $\mathcal{A}(n, 2)$, for all $n \geq 3$.

As the Bruhat order and the secondary Bruhat order coincide on $\mathcal{A}(n, 1)$ and $\mathcal{A}(n, 2)$, we can say that the Bruhat order and the little secondary Bruhat order do not coincide on $\mathcal{A}(n, 1)$ nor $\mathcal{A}(n, 2)$.

We end this section with the characterization of the cover relation for the little secondary Bruhat order. 
Theorem 3.2. Let $A=\left[a_{s, v}\right] \in \mathcal{A}(R, S)$, let $i, j \in\{1, \ldots, m\}$, and let $k, l \in\{1, \ldots, n\}$ such that $A[\{i, j\} ;\{k, l\}]=L_{2}$. Let $A^{\prime}$ be the matrix obtained from $A$ by the interchange $A[\{i, j\} ;\{k, l\}] \rightarrow I_{2}$. Then $A$ covers $A^{\prime}$ in the little secondary Bruhat order if and only if $j=i+1$ and $a_{i, q}=a_{j, q}$, for $k<q<l$.

Proof. If $A$ covers $A^{\prime}$ in the little secondary Bruhat order then, by definition, $j=i+1$. Suppose that there is an integer $q$ with $a_{i, q}=0$ and $a_{i+1, q}=1$. Then $A[\{i, i+1\} ;\{q, l\}]=L_{2}$. Let $C$ be the matrix obtained from $A$ performing the $A[\{i, i+1\} ;\{q, l\}] \rightarrow I_{2}$ interchange. Then $C \prec_{B^{\prime}} A$, and $C[\{i, i+1\} ;\{k, q\}]=L_{2}$. Performing the $C[\{i, i+1\} ;\{k, q\}] \rightarrow I_{2}$ interchange we obtain $A^{\prime}$, and we have a $A^{\prime} \prec{ }_{B^{\prime}} C \prec{ }_{B^{\prime}} A$. Hence $A$ does not cover $A^{\prime}$. A similar argument works when $a_{i, q}=1$ and $a_{i+1, q}=0$.

Conversely, suppose that $j=i+1$ and $a_{i, q}=a_{j, q}$, for $k<q<l$. Then, by Theorem $2.1, A$ covers $A^{\prime}$ in the secondary Bruhat order. This implies that $A$ covers $A^{\prime}$ by the little secondary Bruhat order because, if there is a matrix $D$ such that $A^{\prime} \prec_{B^{\prime}} D \prec_{B^{\prime}} A$, then $A^{\prime} \prec_{\widehat{B}} D \prec_{\widehat{B}} A$.

4. The class $\mathcal{A}(\boldsymbol{n}, \mathbf{2})$. Since the Bruhat order and the secondary Bruhat order coincide on $\mathcal{A}(n, 2)$, Brualdi and Hwang characterized the minimal matrices of $\mathcal{A}(n, 2)$ in the secondary Bruhat order [3].

Theorem 4.1 ([3]). Let $n$ be a positive integer, $n \geq 2$. A matrix $A \in \mathcal{A}(n, 2)$ is minimal in the Bruhat order if and only if $A$ is the direct sum of matrices equal to

$$
J_{2}=\left[\begin{array}{ll}
1 & 1 \\
1 & 1
\end{array}\right] \quad \text { and } \quad F_{3}=\left[\begin{array}{lll}
1 & 1 & 0 \\
1 & 0 & 1 \\
0 & 1 & 1
\end{array}\right]
$$

In this section, we will describe the matrices of $\mathcal{A}(n, 2)$ that are minimal in the little secondary Bruhat order. We start with a result valid in any class $\mathcal{A}(R, S)$.

TheOREm 4.2. Let $A \in \mathcal{A}(R, S)$ be a minimal matrix in the little secondary Bruhat order. Then every leading submatrix of $A$ has nonincreasing row sum vector.

Proof. Let $A=\left[a_{s, v}\right] \in \mathcal{A}(R, S)$ be a minimal matrix in the little secondary Bruhat order and $R=$ $\left(r_{1}, \ldots, r_{m}\right)$. Assume that there is a leading submatrix of $A$, and two integers $i$ and $j$ such that the submatrix $M_{j}=A[\{i, i+1\} ;\{1, \ldots, j\}]$ has

$$
\sum_{t=1}^{j} a_{i, t}<\sum_{t=1}^{j} a_{i+1, t}
$$

Let $j_{0}$ be the smallest integer such that $M_{j_{0}}$ satisfies last conditions. Then $a_{i, j_{0}}=0$ and $a_{i+1, j_{0}}=1$. As $r_{i} \geq r_{i+1}$ then there is an integer $k$, with $k>j_{0}$, such that $a_{i, k}=1$ and $a_{i+1, k}=0$. Therefore, $A$ is not minimal in the little secondary Bruhat order because $A\left[\{i, i+1\} ;\left\{j_{0}, k\right\}\right]=L_{2}$.

The next results are easy consequences of Theorem 4.2 .

Corollary 4.3. Let $A \in \mathcal{A}(R, S)$ be a minimal matrix in the little secondary Bruhat order. Then the first column of $A$ is $\left[\begin{array}{llllll}1 & \ldots & 1 & 0 & \ldots & 0\end{array}\right]^{T}$.

Corollary 4.4. Let $A \in \mathcal{A}(R, S)$ be a minimal matrix in the little secondary Bruhat order. Then the last column of $A$ is $\left[\begin{array}{llllll}0 & \ldots & 0 & 1 & \ldots & 1\end{array}\right]^{T}$.

COROLlary 4.5. Let $n$ be a positive integer. Then the minimal matrices of $\mathcal{A}(n, 1)$ in the Bruhat order, in the secondary Bruhat order, and in the little secondary Bruhat order coincide. Moreover, the matrix $I_{n}$ is the unique minimal matrix of $\mathcal{A}(n, 1)$ in these three orders. 
Corollary 4.6. Let $n$ be a positive integer. Then the minimal matrices of $\mathcal{A}(n, n-1)$ in the Bruhat order, in the secondary Bruhat order, and in the little secondary Bruhat order coincide. Moreover, the matrix $J_{n}-L_{n}$ is the unique minimal matrix of $\mathcal{A}(n, n-1)$ in these three orders.

Note that if $A$ is a minimal matrix in the secondary Bruhat order, then it is also a minimal matrix in the little secondary Bruhat order. In fact, if $A$ is not a minimal matrix in the little secondary Bruhat order, then there is a matrix $C$ such that $C \prec_{B^{\prime}} A$. Therefore, $C \prec_{\widehat{B}} A$ and $A$ would not be a minimal matrix in the secondary Bruhat order. Hence, we may conclude that if a matrix of $\mathcal{A}(R, S)$ is minimal in the secondary Bruhat order, that is, if this matrix does not have a submatrix equal to $L_{2}$, then it is also minimal in the little secondary Bruhat order. However, as we will see, there are matrices of $\mathcal{A}(n, 2)$ that are minimal in the little secondary Bruhat order but not in the secondary Bruhat order.

Let $h$ be an odd positive integer, $h \geq 3$ and $t=\left\lceil\frac{h}{2}\right\rceil$. We define by $V_{h} \in \mathcal{A}(h, 2)$ as follows:

$$
\begin{aligned}
& V_{h}[\{1, \ldots, h\} ;\{1, \ldots, t-1\}]=\left[\begin{array}{cccc}
1 & 0 & \ldots & 0 \\
1 & 0 & \ldots & 0 \\
0 & 1 & \ldots & 0 \\
0 & 1 & \ldots & 0 \\
\vdots & \vdots & & \vdots \\
0 & 0 & \ldots & 1 \\
0 & 0 & \ldots & 1 \\
0 & 0 & \ldots & 0
\end{array}\right] \\
& V_{h}[\{1, \ldots, h\} ;\{t\}]=\left[\begin{array}{lllll}
1 & 0 & \ldots & 0 & 1
\end{array}\right]^{T} \text {, } \\
& V_{h}[\{1, \ldots, h\} ;\{t+1, \ldots, h\}]=\left[\begin{array}{cccc}
0 & 0 & \ldots & 0 \\
1 & 0 & \ldots & 0 \\
1 & 0 & \ldots & 0 \\
0 & 1 & \ldots & 0 \\
0 & 1 & \ldots & 0 \\
\vdots & & \vdots & \\
0 & 0 & \ldots & 1 \\
0 & 0 & \ldots & 1
\end{array}\right] .
\end{aligned}
$$

For instance,

$$
V_{3}=\left[\begin{array}{lll}
1 & 1 & 0 \\
1 & 0 & 1 \\
0 & 1 & 1
\end{array}\right]=F_{3} \text { and } V_{7}=\left[\begin{array}{ccccccc}
1 & 0 & 0 & 1 & 0 & 0 & 0 \\
1 & 0 & 0 & 0 & 1 & 0 & 0 \\
0 & 1 & 0 & 0 & 1 & 0 & 0 \\
0 & 1 & 0 & 0 & 0 & 1 & 0 \\
0 & 0 & 1 & 0 & 0 & 1 & 0 \\
0 & 0 & 1 & 0 & 0 & 0 & 1 \\
0 & 0 & 0 & 1 & 0 & 0 & 1
\end{array}\right]
$$

REMARK 4.7. The matrix $V_{h}, h \geq 3$ does not have any submatrix equal to $L_{2}$ in two consecutive rows. In fact, if

$$
V_{h}[\{i, i+1\} ;\{l\}]=\left[\begin{array}{l}
0 \\
1
\end{array}\right]
$$


for some $1 \leq i, l<n$, then for any $k>l$

$$
V_{h}[\{i, i+1\} ;\{k\}]=\left[\begin{array}{l}
1 \\
1
\end{array}\right] \quad \text { or } \quad V_{h}[\{i, i+1\} ;\{k\}]=\left[\begin{array}{l}
0 \\
0
\end{array}\right]
$$

THEOREM 4.8. Let $n$ be a positive integer, $n \geq 2$. A matrix $A \in \mathcal{A}(n, 2)$ is minimal in the little secondary Bruhat order if and only if $A$ is the direct sum of matrices equal to $J_{2}$ and $V_{h}$, with $h \geq 3$ an odd integer.

Proof. If the matrices $J_{2}$ and $V_{h}$, with $h \geq 3$ an odd integer, do not contain any submatrix equal to $L_{2}$ formed by two consecutive rows, then they are minimal in the little secondary Bruhat order. So, the direct sum of these matrices is also a minimal matrix in the little secondary Bruhat order.

Conversely, let $A=\left[a_{i, j}\right] \in \mathcal{A}(n, 2)$ be a minimal matrix in the little secondary Bruhat order. By Corollary 4.3 , we have $a_{1,1}=a_{2,1}=1$. If $A=\left[a_{i, j}\right] \in \mathcal{A}(n, 2)$, then

$$
A=\left[\begin{array}{cc}
1 & a_{1,2} \\
1 & a_{2,2} \\
0 & \\
\vdots & \\
0 &
\end{array}\right]
$$

If $a_{1,2}=1$ and $a_{2,2}=1$, then $A=J_{2} \oplus A^{\prime}$ where $\oplus$ denotes the direct sum of matrices and $A^{\prime} \in \mathcal{A}(n-2,2)$ is a minimal matrix in the little secondary Bruhat order.

If $a_{1,2}=1$ and $a_{2,2}=0$, then by Theorem 4.2 we get $a_{3,2}=1$. We are going to prove that $a_{2,3}=1$. Assume that $a_{2,3}=0$. Using Theorem 4.2 , we get $a_{3,3}=0, a_{4,3}=1$ and

$$
A=\left[\begin{array}{ccc}
1 & 1 & 0 \\
1 & 0 & 0 \\
0 & 1 & 0 \\
0 & 0 & 1 \\
\vdots & & \\
0 & &
\end{array}\right]
$$

Let $j$ be the integer, $j>3$, such that $a_{2, j}=1$. If $a_{3, j}=0$, then

$$
A[\{2,3\} ;\{2, j\}]=L_{2}
$$

Impossible. Consequently, $a_{3, j}=1$. If $a_{2, j}=a_{3, j}=1, A \in \mathcal{A}(n, 2)$, then $a_{4, j}=0$ and $A[\{3,4\} ;\{3, j\}]=L_{2}$. Impossible. Thus, $a_{2,3}=1$. As $a_{3,2}=1$ and $A[\{2,3\} ;\{2,3\}] \neq L_{2}$ we have $a_{3,3}=1$. Therefore, $A=V_{3} \oplus A^{\prime}$ where $\oplus$ denotes the direct sum of matrices and $A^{\prime} \in \mathcal{A}(n-3,2)$ is a minimal matrix in the little secondary Bruhat order.

If $a_{1,2}=0$ then, by Theorem 4.2, we get $a_{2,2}=0$ and $a_{3,2}=a_{4,2}=1$. Let $j$ be the integer, $j>2$, such that $a_{1, j}=1$. By Theorem 4.2 , if $k$ is the integer such that $a_{2, k}=1, k>1$, then $k \geq j$. Suppose that $k=j$ then we have $a_{3, j}=0$ and $A[\{2,3\} ;\{2, j\}]=L_{2}$, which is impossible. So, $k>j$. Let $l$ and $g$ be the integers, 
$l, g>2$, such that $a_{3, l}=1=a_{4, g}$. Again by Theorem 4.2, $g \geq l \geq k$. In this way, using Theorem 4.2, we conclude that

$$
A[\{1,2, \ldots, 2 j-1\} ;\{1, \ldots, j\}]=\left[\begin{array}{ccccc}
1 & 0 & \ldots & 0 & 1 \\
1 & 0 & \ldots & 0 & 0 \\
0 & 1 & \ldots & 0 & 0 \\
0 & 1 & \ldots & 0 & 0 \\
\vdots & \vdots & & \vdots & \vdots \\
0 & 0 & \ldots & 1 & 0 \\
0 & 0 & \ldots & 1 & 0 \\
0 & 0 & \ldots & 0 & 1
\end{array}\right]
$$

As $a_{1,1}=a_{1, j}=1$ then $a_{1, f}=0$, for $3 \leq f \leq n$. By Theorem 4.2, we get

$$
A[\{1,2, \ldots, 2 j-1\} ;\{j+1, \ldots, 2 j-1\}]=\left[\begin{array}{ccccc}
0 & 0 & \ldots & 0 & 0 \\
1 & 0 & \ldots & 0 & 0 \\
1 & 0 & \ldots & 0 & 0 \\
0 & 1 & \ldots & 0 & 0 \\
\vdots & \vdots & & \vdots & \vdots \\
0 & 0 & \ldots & 1 & 0 \\
0 & 0 & \ldots & 0 & 1 \\
0 & 0 & \ldots & 0 & 1
\end{array}\right] .
$$

Therefore, $A=V_{2 j-1} \oplus A^{\prime}$ where $\oplus$ denotes the direct sum of matrices and $A^{\prime} \in \mathcal{A}(n-(2 j-1), 2)$ is a minimal matrix in the little secondary Bruhat order. The theorem now follows by the induction on $n$.

5. The class $\mathcal{A}(\boldsymbol{n}, \boldsymbol{k})$. In [4], the authors showed that if $n$ and $k$ are integers with $0 \leq k \leq n$, and $\mathcal{A}(n, k) \neq \emptyset$, then the class $\mathcal{A}(n, k)$ has a unique minimal element in the secondary Bruhat order if and only if $k=0,1, n-1, n$ or $n=2 k$. Moreover, they proved that the unique minimal matrix in $\mathcal{A}(2 k, k)$ is $J_{k} \oplus J_{k}$, where $\oplus$ denotes the direct sum of matrices, and the unique minimal matrix in $\mathcal{A}(n, n-1)$ is $J_{n}-L_{n}$. If $\mathcal{A}(n, 0)$ and $\mathcal{A}(n, n)$ each contain only one matrix, then trivially they have a unique minimal matrix in the little secondary Bruhat order. Using Corollaries 4.5 and 4.6 we get $\mathcal{A}(n, k)$, with $k=1$ or $n-1$, has a unique minimal matrix in the little secondary Bruhat order. Moreover, as we mentioned in the last section, if $A \in \mathcal{A}(n, k)$ is a minimal matrix in the secondary Bruhat order, then it is also a minimal matrix in the little secondary Bruhat order. So, if $k \notin\{0,1, n-1, n\}, n \neq 2 k$ and $\mathcal{A}(n, k) \neq \emptyset$, then $\mathcal{A}(n, k)$ has at least two minimal matrices in the little secondary Bruhat order. In the next theorem, we show that the families of classes $\mathcal{A}(n, k)$ with a unique minimal element coincide in the secondary Bruhat order and in the little secondary Bruhat order.

Theorem 5.1. Let $n$ and $k$ be integers with $0 \leq k \leq n$ and $\mathcal{A}(n, k) \neq \emptyset$. Then the class $\mathcal{A}(n, k)$ has a unique minimal element in the little secondary Bruhat order if and only if $k=0,1, n-1, n$ or $n=2 k$. Moreover, the unique minimal matrix in $\mathcal{A}(2 k, k)$ is $J_{k} \oplus J_{k}$.

Proof. From last observations, we only need to show that the unique minimal matrix in the little secondary Bruhat order on $\mathcal{A}(2 k, k)$ is $J_{k} \oplus J_{k}$. Using Corollary 4.5 and Theorem 4.8, last sentence is valid for $k=1,2$. Suppose there is an integer $k, k \geq 3$, such that $\mathcal{A}(2 k, k)$ contains a matrix $A=\left[a_{i, j}\right] \neq J_{k} \oplus J_{k}$ which is minimal in the little secondary Bruhat order. 
Let $l$ be the smallest integer such that $a_{k, l}=0$. By Corollary 4.3, we conclude that $l>1$. Moreover,

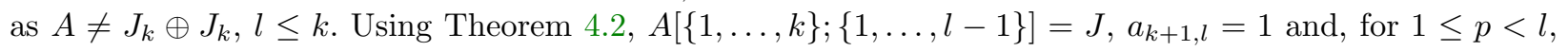
$a_{k+1, p}=0$. As each row of $A$ has $k$ ones, there is an integer $f>l$ with $a_{k, f}=1$. If $a_{k+1, f}=0$, then $A[\{k, k+1\} ;\{l, f\}]=L_{2}$ which is impossible because $A$ is minimal. So, $a_{k+1, f}=1$ and

$$
A[\{k, k+1\} ;\{1, \ldots, l-1, l, f\}]=\left[\begin{array}{ccccc}
1 & \ldots & 1 & 0 & 1 \\
0 & \ldots & 0 & 1 & 1
\end{array}\right] .
$$

Using Corollary 4.4, we get $A[\{k, k+1\} ;\{2 k\}]=\left[\begin{array}{l}0 \\ 1\end{array}\right]$. As row $k$ of $A$ has $k$ ones, repeating last argument, there are $k-(l-1)$ integers $f_{1}, \ldots, f_{k-(l-1)}$, with $l<f_{1}<\cdots<f_{k-(l-1)}<2 k$, such that the submatrix of $A$ restricted to these columns and to rows $k$ and $k+1$ is

$$
A\left[\{k, k+1\} ;\left\{l, f_{1}, \ldots, f_{k-(l-1)}, 2 k\right\}\right]=\left[\begin{array}{ccccc}
0 & 1 & \ldots & 1 & 0 \\
1 & 1 & \ldots & 1 & 1
\end{array}\right] .
$$

As row $k+1$ of $A$ has $k$ ones and the last matrix has $(k-(l-1))+2=k-l+3$ ones in the second row, $l \geq 3$. Moreover, if $l>3$, then there are $l-3$ integers $h_{1}, \ldots, h_{l-3}$, with $l<h_{1}<\cdots<h_{l-3}<2 k$, such that the submatrix of $A$ restricted to these columns and to rows $k$ and $k+1$ is

$$
A\left[\{k, k+1\} ;\left\{l, h_{1}, \ldots, h_{l-3}, 2 k\right\}\right]=\left[\begin{array}{ccccc}
0 & 0 & \ldots & 0 & 0 \\
1 & 1 & \ldots & 1 & 1
\end{array}\right] .
$$

Consequently, we know the elements of $A$ that are in rows $k$ and $k+1$, and in $2(l-1)+(k-(l-1))=$ $k+l-1<2 k$ columns. From the fact that $A[\{k, k+1\} ;\{f\}]=\left[\begin{array}{l}1 \\ 1\end{array}\right]$ we conclude that there is at least one integer $v$, with $l<v<2 k$, such that

$$
A[\{k, k+1\} ;\{v\}]=\left[\begin{array}{l}
0 \\
0
\end{array}\right]
$$

Let $t=f_{k-l+1}$ be the largest integer such that $A[\{k, k+1\} ;\{t\}]=\left[\begin{array}{l}1 \\ 1\end{array}\right]$, and let $g$ be the largest integer such that $A[\{k, k+1\} ;\{g\}]=\left[\begin{array}{l}0 \\ 0\end{array}\right]$.

If $g>t$, using Theorem 4.2 we get $A[\{1, \ldots, k, k+1\} ;\{g\}]=O_{k+1,1}$. Consequently, column $g$ of $A$ will be, at most, $k-1$ ones, which is impossible.

Assume that $g<t$. Then the first row of $A[\{k+1, \ldots, 2 k\} ;\{t, \ldots, 2 k\}]$ is $[11 \ldots 1]$. Assume that there is a integer $s, k<s \leq 2 k$, and an integer $j, t \leq j \leq 2 k$ such that $a_{s, j}=0$. Then

$$
\sum_{i=1}^{t-1} a_{k, i}<\sum_{i=1}^{t-1} a_{s, i}
$$

But this contradicts Theorem 4.2. Then

$$
A[\{k+1, \ldots, 2 k\} ;\{t, \ldots, 2 k\}]=J_{k, 2 k-t+1},
$$

and consequently, the column $t$ of $A$ will have, at least, $k+1$ ones, which is impossible.

Thus, $A=J_{k} \oplus J_{k}$. 
6. Symmetric classes. A matrix obtained from a symmetric $n$-by- $n(0,1)$ matrix by an $L_{2} \rightarrow I_{2}$ interchange may not be a symmetric matrix. Sometimes, we need to perform two or more $L_{2} \rightarrow I_{2}$ interchanges to obtain another symmetric matrix. In the case of the little secondary Bruhat order, the only way to perform an $L_{2} \rightarrow I_{2}$ and obtain a symmetric matrix is doing this interchange in a submatrix of the form $A[\{i, i+1\} ;\{i, i+1\}]$. Since this is very restrict, in this section we will study another partial order relation in classes of symmetric $(0,1)$-matrices. Two symmetric matrices are related by this new partial order relations if one of them is obtained from the other by, at most, two $L_{2} \rightarrow I_{2}$ interchanges perform in two consecutive rows.

Let $R=\left(r_{1}, \ldots, r_{n}\right)$ be a nonincreasing positive integral vector such that $\mathcal{A}(R, R) \neq \emptyset$. We denote by $\mathcal{A}^{S}(R)$ the class of all symmetric matrices of $\mathcal{A}(R, R)$. Similarly, if $n$ and $k$ are positive integers such that $\mathcal{A}(n, k) \neq \emptyset$, the subset of $\mathcal{A}(n, k)$ of all symmetric matrices will be denoted by $\mathcal{A}^{S}(n, k)$.

Let $A, C \in \mathcal{A}^{S}(R)$, we say that $A$ is obtained from $C$ by a symmetric interchange if $A$ and $C$ are equal except in a submatrix which is replaced by another matrix in four possible ways:

$$
A[\{i, i+1, i+2\}]=\left[\begin{array}{ccc}
0 & 1 & 1 \\
1 & 1 & 0 \\
1 & 0 & *
\end{array}\right] \leftrightarrow\left[\begin{array}{lll}
1 & 1 & 0 \\
1 & 0 & 1 \\
0 & 1 & *
\end{array}\right]=C[\{i, i+1, i+2\}] .
$$

The matrix $C[\{i, i+1, i+2\}]$ is obtained from $A[\{i, i+1, i+2\}]$ by the $A[\{i, i+1\} ;\{i, i+2\}]=L_{2} \rightarrow I_{2}$ interchange followed by the $L_{2} \rightarrow I_{2}$ interchange in rows $i+1, i+2$ and columns $i, i+1$.

$$
A[\{i, i+1, i+2\}]=\left[\begin{array}{ccc}
* & 0 & 1 \\
0 & 1 & 1 \\
1 & 1 & 0
\end{array}\right] \leftrightarrow\left[\begin{array}{ccc}
* & 1 & 0 \\
1 & 0 & 1 \\
0 & 1 & 1
\end{array}\right]=C[\{i, i+1, i+2\}] .
$$

The matrix $C[\{i, i+1, i+2\}]$ is obtained from $A[\{i, i+1, i+2\}]$ by the $A[\{i+1, i+2\} ;\{i, i+2\}]=L_{2} \rightarrow I_{2}$ interchange followed by the $L_{2} \rightarrow I_{2}$ interchange in rows $i, i+1$ and columns $i+1, i+2$.

$$
A[\{i, i+1, p, p+1\}]=\left[\begin{array}{cccc}
* & * & 0 & 1 \\
* & * & 1 & 0 \\
0 & 1 & * & * \\
1 & 0 & * & *
\end{array}\right] \leftrightarrow\left[\begin{array}{cccc}
* & * & 1 & 0 \\
* & * & 0 & 1 \\
1 & 0 & * & * \\
0 & 1 & * & *
\end{array}\right]=C[\{i, i+1, p, p+1\}] .
$$

In each case, $*$ denotes an unspecified entry, and in the last case, $i, p$ are appropriated integers with $p>i+1$. In all cases, we say that the matrix on the right is obtained from the matrix on the left by a symmetric $L_{2} \rightarrow I_{2}$ interchange, and the matrix on the left is obtained from the matrix on the right by a symmetric $I_{2} \rightarrow L_{2}$ interchange.

Let $A_{1}, A_{2} \in \mathcal{A}^{S}(R)$, we say that $A_{1}$ precedes $A_{2}$ in the symmetric little secondary Bruhat order, written $A_{1} \preceq_{B^{\prime \prime}} A_{2}$, provided $A_{1}=A_{2}$ or $A_{1}$ can be obtained from $A_{2}$ by a sequence of symmetric $L_{2} \rightarrow I_{2}$ interchanges. 
The purpose of this section is to show a family of matrices in $\mathcal{A}(n, k)$, with $n \geq 4$ and $k \neq 0, n$, where the restriction of the little secondary Bruhat order does not have a unique minimal matrix.

The next result shows us that if $n \geq 3$, then there are at least two minimal matrices in the symmetric little secondary Bruhat order on $\mathcal{A}^{S}(n, 1)$.

TheOREM 6.1. Let $n$ be a positive integer, $n \geq 2$. A matrix $A \in \mathcal{A}^{S}(n, 1)$ is minimal in the symmetric little secondary Bruhat order if and only if there are not integers $i, j, 1 \leq i<n$, and $1 \leq j<n$ such that $A[\{i, i+1\} ;\{j, j+1\}]=L_{2}$ with $j \neq i+1$ and $i \neq j+1$.

Proof. Let $A \in \mathcal{A}^{S}(n, 1)$. Suppose there are integers $i, j, 1 \leq i<n$ and $1 \leq j<n$ such that $A[\{i, i+$ $1\} ;\{j, j+1\}]=L_{2}$ with $j \neq i+1$ and $i \neq j+1$.

If $j=i$, then

$$
A[\{i, i+1\}]=L_{2},
$$

and we can obtain a matrix $C$ from $A$ by the symmetric $L_{2} \rightarrow I_{2}$ interchange, (6.1). Consequently, $A$ is not minimal in the symmetric little secondary Bruhat order.

If $j<i-1$, then

$$
A[\{j, j+1, i, i+1\}]=\left[\begin{array}{cccc}
* & * & 0 & 1 \\
* & * & 1 & 0 \\
0 & 1 & * & * \\
1 & 0 & * & *
\end{array}\right]
$$

where $*$ denotes an unspecified entry, and we can obtain a matrix $C$ from $A$ by the symmetric $L_{2} \rightarrow I_{2}$ interchange (6.4). Consequently, $A$ is not minimal in the symmetric little secondary Bruhat order.

If $j>i$, then

$$
A[\{i, i+1, j, j+1\}]=\left[\begin{array}{cccc}
* & * & 0 & 1 \\
* & * & 1 & 0 \\
0 & 1 & * & * \\
1 & 0 & * & *
\end{array}\right]
$$

where $*$ denotes an unspecified entry, and we can obtain a matrix $C$ from $A$ by the symmetric $L_{2} \rightarrow I_{2}$ interchange (6.4). Consequently, $A$ is not minimal in the symmetric little secondary Bruhat order.

Conversely, suppose that if there are integers $i, j$ such that $1 \leq i<n, 1 \leq j<n$ and $A[\{i, i+1\} ;\{j, j+$ $1\}]=L_{2}$, then $j=i+1$ or $i=j+1$. Let $p, q$ be two integers such that $A[\{p, p+1\} ;\{q, q+1\}]=L_{2}$. Assume that $q=p+1$. Then

$$
A[\{p, q, q+1\}]=\left[\begin{array}{ccc}
* & 0 & 1 \\
0 & 1 & 0 \\
1 & 0 & *
\end{array}\right]
$$

If $p=q+1$, then

$$
A[\{q, p, p+1\}]=\left[\begin{array}{ccc}
* & 0 & 1 \\
0 & 1 & 0 \\
1 & 0 & *
\end{array}\right],
$$

where $*$ denotes an unspecified entry. So, $A$ does not contain any symmetric $L_{2} \rightarrow I_{2}$ interchange (6.1) or (6.4). Thus, $A$ is minimal in the symmetric little secondary Bruhat order. 
Electronic Journal of Linear Algebra, ISSN 1081-3810

EXAMPLE 6.2. Using last result, the minimal matrices in the symmetric little secondary Bruhat order on $\mathcal{A}^{S}(3,1)$ are

$$
I_{3} \text { and } L_{3}=\left[\begin{array}{ccc}
0 & 0 & 1 \\
0 & 1 & 0 \\
1 & 0 & 0
\end{array}\right]
$$

The following matrix is minimal in the symmetric little secondary Bruhat order on $\mathcal{A}^{S}(7,1)$

$$
\left[\begin{array}{lllllll}
0 & 0 & 1 & 0 & 0 & 0 & 0 \\
0 & 0 & 0 & 0 & 0 & 0 & 1 \\
1 & 0 & 0 & 0 & 0 & 0 & 0 \\
0 & 0 & 0 & 0 & 0 & 1 & 0 \\
0 & 0 & 0 & 0 & 1 & 0 & 0 \\
0 & 0 & 0 & 1 & 0 & 0 & 0 \\
0 & 1 & 0 & 0 & 0 & 0 & 0
\end{array}\right]
$$

Let $s$ be a positive integer and $i, j \in\{1, \ldots, s\}$. Denote by $E_{i, j}$ the $s$-by-s matrix with entry $(i, j)$ equal to one and all other entries equal to zero.

Corollary 6.3. Let $n$ be a positive integer, $n \geq 3$. Let $P_{n}$ be the matrix obtained from $I_{n}$ by the symmetric $I_{n}[\{1, n\}]=I_{2} \rightarrow L_{2}$ interchange (6.4). The matrices $I_{n}$ and $P_{n}$ are minimal matrices in the symmetric little secondary Bruhat order on $\mathcal{A}^{S}(n, 1)$.

Proposition 6.4. Let $n$ be a positive integer, $n \geq 3$. Let $Q_{n}$ be the matrix obtained from $I_{n}$ by the symmetric $\left(J_{n}-L_{n}\right)[\{1, n\}]=I_{2} \rightarrow L_{2}$ interchange (6.4). The matrices $J_{n}-L_{n}$ and $Q_{n}$ are minimal matrices in the symmetric little secondary Bruhat order on $\mathcal{A}^{S}(n, n-1)$.

Proof. Since $n \geq 3$, none of the matrices $J_{n}-L_{n}$ and $Q_{n}$ has a symmetric $L_{2} \rightarrow I_{2}$ interchange (6.1), (6.2), (6.3), (6.4). So, they are minimal matrices.

ThEOREM 6.5. Let $n$ and $k$ be integers with $2 \leq k \leq n$ and $\mathcal{A}^{S}(n, k) \neq \emptyset$. Then the class $\mathcal{A}^{S}(n, k)$ has a unique minimal element in the symmetric little secondary Bruhat order if and only if $k=0, n$.

Proof. Since each of the classes $\mathcal{A}^{S}(n, 0)$ and $\mathcal{A}^{S}(n, n)$ has one matrix, trivially this matrix is minimal in the symmetric little secondary Bruhat order.

Conversely, using Theorem 5.1, Corollary 6.3, and Proposition 6.4 we only need to show that if $\mathcal{A}^{S}(2 k, k)$ $\neq \emptyset$, then it has at least two minimal matrices in the symmetric little secondary Bruhat order. Moreover, as the matrix $J_{k} \oplus J_{k}$ is minimal in the little secondary Bruhat order on $\mathcal{A}(2 k, k)$ and is symmetric, then it is minimal in the symmetric little secondary Bruhat order on $\mathcal{A}^{S}(2 k, k)$.

Consider the matrix $D_{2 k}$ obtained from $J_{k} \oplus J_{k}$ by the symmetric $\left(J_{k} \oplus J_{k}\right)[\{1,2 k\}]=I_{2} \rightarrow L_{2}$ interchange (6.4). If $D_{2 k}$ does not have any symmetric $L_{2} \rightarrow I_{2}$ interchange (6.1), (6.2), (6.3), (6.4) and it is symmetric then it is a minimal matrix. So, $\mathcal{A}^{S}(2 k, k)$ has at least two minimal matrices in the symmetric little secondary Bruhat order.

7. Conclusions. We have studied the minimal matrices in the little secondary Bruhat order on the class of $(0,1)$-matrices. We have described its cover relation. We have characterized the minimal matrices in this order on classes of $(0,1)$-matrices with constant row and column sum $k$, when $k=1,2$. We also presented 
a necessary and sufficient condition on $k$ and on the size of the matrices to have classes of $(0,1)$-matrices with a unique minimal element in the little secondary Bruhat order.

A variant of the little secondary Bruhat order in classes of symmetric $(0,1)$-matrices with constant row sum $k$ was studied. We showed that, in general, these classes have many minimal matrices with respect to this partial order.

We conclude with some questions that we have not yet considered in much detail.

1. Let $q$ be a positive integer. Investigate other partial orders on $\mathcal{A}(R, S)$ :

$A_{1}$ precedes $A_{2}$ if and only if $A_{1}=A_{2}$ or $A_{2}$ can be transformed into $A_{1}$ by a sequence of $L_{2} \rightarrow I_{2}$ interchanges where in each such interchange, $L_{2}$ is a submatrix formed by two rows $i$ and $j$, with $i<j$, such that $j-i \leq q$.

2. Let $q$ and $p$ be positive integer. Investigate other partial orders on $\mathcal{A}(R, S)$ :

$A_{1}$ precedes $A_{2}$ if and only if $A_{1}=A_{2}$ or $A_{2}$ can be transformed into $A_{1}$ by a sequence of $L_{2} \rightarrow I_{2}$ interchanges where in each such interchange, $L_{2}$ is a submatrix formed by two rows $i$ and $j$, with $i<j$, such that $j-i \leq q$ and by two columns $l$ and $k$, with $l<k$, such that $k-l \leq p$.

3. Let $q$ and $p$ be positive integer. Investigate other partial orders on $\mathcal{A}(R, S)$ :

$A_{1}$ precedes $A_{2}$ if and only if $A_{1}=A_{2}$ or $A_{2}$ can be transformed into $A_{1}$ by a sequence of $L_{2} \rightarrow I_{2}$ interchanges where in each such interchange, $L_{2}$ is a submatrix formed by two rows $i$ and $j$, with $i<j$, such that $j-i \leq q$ or by two columns $l$ and $k$, with $l<k$, such that $k-l \leq p$.

4. Study the relation between the orders described before.

Note that these relations are partial order relations because if two matrices are related by one of these relations, then they are also related by the Bruhat order.

\section{REFERENCES}

[1] R.A. Brualdi. Matrices of zeros and ones with fixed row and column-sum vectors. Linear Algebra Appl., 33:159-231, 1980.

[2] R.A. Brualdi and S.-G. Hwang. A Bruhat order for the class of $(0,1)$-matrices with row sum vector $R$ and column sum vector S. Electron. J. Linear Algebra, 12:6-16, 2004.

[3] R.A. Brualdi. Combinatorial Matrix Classes. Encyclopedia of Mathematics and its Applications, vol. 108. Cambridge University Press, Cambridge, 2006.

[4] R.A. Brualdi and L. Deaett. More on the Bruhat order for (0,1)-matrices. Linear Algebra Appl., 421:219-232, 2007.

[5] R.A. Brualdi and E. Fritscher. Bruhat order of tournaments. Linear Algebra Appl., 458:261-279, 2014.

[6] R.A. Brualdi and G. Dahl. Doubly stochastic matrices and the Bruhat order. Czechoslov. Math. J. 66(141):681-700, 2016.

[7] R.A. Brualdi and M.W. Schroeder. Alternating sign matrices and their Bruhat order. Disc. Math., 340(8):1996-2019, 2017.

[8] R.A. Brualdi, R. Fernandes, and S. Furtado. On the Bruhat order of labeled graphs. Disc. Appl. Math., 258:49-64, 2019.

[9] A. Conflitti, C.M. Fonseca, and R. Mamede. The maximal length of a chain in the Bruhat order for a class of binary matrices. Linear Algebra Appl., 436:753-757, 2012.

[10] A. Conflitti, C.M. da Fonseca, and R. Mamede. On the largest size of an antichain in the Bruhat order for A(2k,k). Order, 30:255-260, 2013.

[11] H.F. Cruz, R. Fernandes, and S. Furtado. Minimal matrices in the Bruhat order for symmetric $(0,1)$-matrices. Linear Algebra Appl., 530:160-184, 2017.

[12] R. Fernandes, H.F. da Cruz, and D. Salomão. Classes of $(0,1)$-matrices where the Bruhat Order and the Secondary Bruhat Order Coincide. Order, 37:207-221, 2020.

[13] R. Fernandes, H.F. Cruz, and D. Salomão. Latin Squares and their Bruhat Order. Contrib. Disc. Math., 15:102-120, 2020.

[14] R. Fernandes, H.F. Cruz, and D. Salomão. The Bruhat order on classes of isotopic Latin squares. Portugaliae Mathematica, 77:111-131, 2020.

[15] R. Fernandes, H.F. Cruz, and D. Salomão. On a conjecture concerning the Bruhat order. Linear Algebra Appl. 600:82-95, 2020. 
[16] R. Fernandes and S. Furtado. Extremal matrices for the Bruhat-graph order. Linear Multilinear Algebra, 2020. DOI:10.1080/03081087.2020.1749540.

[17] R. Fernandes and H.F. da Cruz. On the term rank partition. Linear Algebra Appl. 458:134-148, 2014.

[18] R. Fernandes, H.F. da Cruz, and S.C. Palheira. Matrices in $\mathcal{A}(R, S)$ with minimum t-term ranks. Linear Algebra Appl. 585:239-261, 2020.

[19] D. Gale. A theorem on flows in networks. Pacific J. Math., 7:1073-1082, 1957.

[20] M. Ghebleh. On maximum chains in the Bruhat order of $\mathcal{A}(n, 2)$. Linear Algebra Appl., 446:377-387, 2014.

[21] M. Ghebleh. Antichains on (0,1)-matrices through invertions. Linear Algebra Appl., 458:503-511, 2014

[22] H.J. Ryser. Combinatorial properties of matrices of zeros and ones. Can. J. Math., 9:371-377, 1957. 